\author{
Military Technical College \\ Kobry El-Kobbah, \\ Cairo, Egypt
}

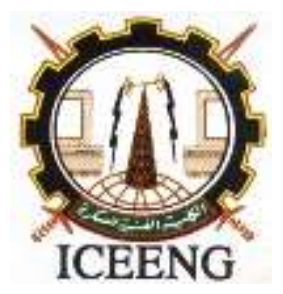

\author{
$11^{\text {th }}$ International Conference \\ on Electrical Engineering \\ ICEENG 2018
}

\title{
Distance and Error Measurement for Wireless Sensor Network System Localization Using RSSI Measurement
}

\author{
S. M. Abd-ElKhaliq *, M. Faied *, R. A. Aboul Seoud *, A. M. Gody *
}

\begin{abstract}
Wireless sensor network is used to detect the change in the copper cables and fiber cables pipelines environment in order to identify and prevent faults and failures in various systems. This paper focuses on communication cables pipelines that span a few kilometers (1-2 km). Those cables and their tracks are buried under the ground for a very long time without maintenance. Communication devices are not fully functioning in high temperature environment. Also, moisture could increase water ratio in the surrounding soil causing problems in these communication cables pipelines functionality. In case of problem occurrence, actions are taken to solve the problem after its existence. To reach the target , we use a network of wireless sensors to detect the changes in the surrounding atmosphere then transmit these changes to a base station (sink) located far away from the remote site. The location of the blind node (nodes that are not aware with their position in the grid of the network) among the other nodes is automatically determined. By calculating the Received Signal Strength Indicator - RSSI of the sensor nodes. The collected data and position estimated techniques are being validated. The results showed an acceptable localization error as the error in the node location was $0.74 \mathrm{~m}$ and when the anchor nodes increased from three to four nodes, the localization error decreases from $0.74 \mathrm{~m}$ to $0.56 \mathrm{~m}$, this means that the localization error decreases by $0.18 \mathrm{~m}$ when add one more anchor node. The proposed technique offers the requirements of accuracy with simple hardware, without complex timing also with less bandwidth sensitive and low cost which greatly enhance the scope of localization site-based applications.
\end{abstract}

\section{KEY WORDS}

Localization System, Received Signal Strength Indicator (RSSI), Wireless Sensor Network (WSN).

${ }^{*}$ Dept.of Electrical Engineering, Fayoum University, Fayoum, Egypt.

\section{Introduction}


Wireless sensor network (WSNs) is classified as an important technology in this century [7]. Thanks to recent developments in the micro electromechanical system (MEMS) and wireless communication technologies, they provide small, cheap and smart sensor that is deployed in a physical area and via wireless network connections. The wireless network has a lot of variety of civilian and military applications, for example, environmental applications monitoring and controlling industry process and control of oil pipelines and gas pipelines, monitoring medical patient surveillance control of the battlefield, [8]. If the sensor nodes are well defined, they can operate independently to measure the temperature, luminosity and moisture and so on. The sensors in the copper cables and fiber cables pipeline are used to sense temperature, vibrations and humidity. The sensor nodes send the collected (sensed) data to the sink node, as the sink node collects the data from all nodes. The sensors can be deployed everywhere in the site-based services as sensor sites will be important. Thanks to the uniqueness of traditional wireless communication networks, for example, cellular networks and mobile communication networks (MANET mobile ad hoc network), wireless networks (WSNs) are characterized by unique characteristics, for example, increasing the intensity of node propagation, unreliable sensor nodes, and severe limitations on energy, computing and storage [3] that presents many new challenges in the development and the application of WSNs. The network usually consists of a large number of low-cost, low-capacity and multifunctional sensor nodes deployed in an area of interest [3].

This compact sensor (node) is small in size but is supported with built in microprocessors, and radio transceivers, so they have sensory ability, as well as data processing and communication capabilities

The Sensors communicate across a short distance through wireless medium and they work together to accomplish a specific task. The site where the sensors are located is very important, because the data collected from the sensor does not make sense if the site where the sensors are located is unknown in the application. The methods that use ultrasonic or laser waves can achieve high accuracy, but adding a device to the location adds to the size, cost, and energy requirements. For these reasons, these methods are not suitable for sensor networks. An inexpensive wireless frequencybased approach has been studied with low configuration requirements [3, 4]. Studies have shown that the received signal strength indicator (RSSI) has a greater variation because it is affected by the shading and fading. Thus, the RSSI-based approach needs more data from other methods to achieve higher accuracy [5].

\section{Localization System Measurement Techniques}

\section{1- Measurements using Angle-of-Arrival:}

(AOA) is the angel of arrival measurements obtained by applying two basic methods,

I. Using the amplitude response of the receiver antenna

II. The phase of response to the receiver antenna.

The measurement of angle of arrival techniques using the phase interferometry are based on the phase-out differences in the arriving front wave. The amplitude is measured in the antenna of the receiver. The variation in the reception pattern affects the amplitude response of the antenna. To apply this method, a variety of antennas are used. The sensitivity and the width of the beam are responsible for obtaining the measurement accuracy. The angle of arrival measurements depend on the route of the 
Line Of Sight (LOS) between the transmitter and the receiver. Other factors influenced the measurements: multiple paths and multiple shading. The multi-path signal appears as another signal that comes from a completely different direction, thus causing a large error in measuring the angle of arrival. The definition of $A O A$ is as the angle between the incident wave and the propagation direction, this known as orientation.

Orientation is a fixed direction that the AOAs are measured. The absolute value of $A O A$ is 0 degrees when it is oriented to the north direction. AOA can be measured by using an array of antennas on each sensor node.

Shown in Fig. 1if the orientation of the unknown node is known then we have:

$\theta 1$ : AOA of the signal sent from node b1 to node $U$

$\theta 2$ : AOA of the signal sent from node b2 to node $U$

$\Delta \theta$ : the orientation of the $U$

\subsection{Measurements using Time of Arrival (TOA)}

Another type of localizing is the Time of Arrival (TOA) this technique measures the taken time of the signal to reach the receiver. This is done by calculating the time the mobile phone responds to the investigation or the instructions sent to the mobile phone from the base station. The total elapsed time from the moment the signal was sent to the moment the mobile response is detected, consisting of a total signal delay of roundtrip and any processing delay and response within the mobile unit.

In Fig. 2 If the TOA estimations is known as $T_{1}, T_{2}$ and $T_{3}$, then the TDOA estimations are $t_{21}$ and $t_{23}$ can be estimated in accordance with the following equations:

$$
\begin{array}{lll}
t_{21}=T_{1}-T_{2} & , & d_{21}=t_{21} v \\
t_{23}=T_{3}-T_{2}, & d_{23}=t_{23} v
\end{array}
$$

where, $v$ is wave speed, $t_{m}$ is the time difference of a received wave

In this way, TOAs that is transmitted from the target can be measured by three or more sensors, each one of them makes circles and intersections give the target location.

\subsection{Measurements using Time-Difference-of-Arrival:}

The short form is TDOA, this is another way between receivers to measure the difference in the signal arrival times. The basic idea in this method is the time difference. There is a synchronized time for the receivers this is the way that the receiver times are compared. Estimation techniques used to measure time are the general way in which calculating the difference in time between the measurements of the receiver from two primary stations to produce a relative TDOA. If the receiver receives an identical signal, but at a different time, the high-level relationship indicator goes beyond the time of delay when it is measured by a different time difference. By measuring the TDOA that emanate from three or more simultaneous sender with a well-known site, it helps a single receiver to find its location.

The principle of measuring the difference in time in the hyperbolic mode is set out in Fig. 3 the hyperbolic is an imaginary set of points in the constant range- difference $\left(\mathrm{C}_{0} \Delta t\right)$ of two sensors and each pair of sensors gives a hyperbola that the transmitter lies on. The intersection of hyperbolas is the estimate of the location. 


\subsection{Measurement Using Received Signal Strength Indicator (RSSI)}

Measurement Using Received Signal Strength Indicator (RSSI) is widely used in localization [3],[11],[13],[14]; this technique concentrates on measuring the power of the Received Signal, based on knowing the transmission power the effective loss in propagation calculated. Translating this loss into distance estimation can be done by theoretical and experimental models. This method is used mainly for RF signals. The strength of the receiving signal is based on power monitoring. In this method, the sensor estimates the received signal strength by combining the signal power of a particular frequency range as an estimate of the energy of the receiving signal. By evaluating the transmitting power a RSS antenna gives a wide range of information. Therefore, RSSI is a measure of the strength of the radio signal in its receiver, and we can also say an indication of the level of power that the antenna receives.

RSSI is used in the range-based application with a predefined bandwidth. The frequency of the radio signal emitted by the transmitter and the gain of the antenna is considered to obtain the appropriate information of RSSI.

The RSSI method describes the relation between the receiver power and the transmitted power of the wireless signal and the distance between the nodes

$$
\begin{aligned}
P_{r} & =P_{t}\left(\frac{1}{d}\right)^{n} \\
10 \log P_{r} & =10 \log P_{t}-10 n \log d
\end{aligned}
$$

$P_{r}$ is the received power

$P_{t}$ is the transmitted power

$D$ : distance between sending and receiving nodes

10logP: An expression of power converted to $\mathrm{dBm}$

$$
P_{r}(d B m)=A-10 n \log d
$$

So the relationship between RSSI and d can be defined by $A$ and $n$ parameters ( $A$ is the received signal strength at one meter distance)\& ( $n$ is the path loss exponent). In the collection phase, the most popular methods are:

\section{The Methods of Calculation Of The Blind Node Location}

\section{1 - Hyperbolic Trilateration}

The basic formula of the general equation of a sphere is given by equation (6) (the center of the sphere is the origin point);

$$
d^{2}=x^{2}+y^{2}+z^{2}
$$

Where, $d$ is the radius of the sphere from a point lying on the sphere with dimensions $(\mathrm{x}, \mathrm{y}, \mathrm{z})$

If the center of a sphere is at the point $\left(\mathrm{x}_{\mathrm{a}}, \mathrm{y}_{\mathrm{a}}, \mathrm{z}_{\mathrm{a}}\right)$ the equation is simplified as in equation (7) and the blind node is at the point ( $x, y, z)$;

$$
d^{2}=\left(x-x_{a}\right)^{2}+\left(y-y_{a}\right)^{2}+\left(z-z_{a}\right)^{2}
$$


We will assume that the nodes are on the same plane, then consider the three anchor nodes $(a, b$ and $c)$ that has the distance $\left(d_{a}, d_{b}, d_{c}\right)$ from the blind node as in Fig.4; To calculate the radius of the spheres as shown below:

Sphere A ;

Sphere B ;

$$
d_{a}^{2}=\left(x-x_{a}\right)^{2}+\left(y-y_{a}\right)^{2}
$$

Sphere C;

$$
d_{b}^{2}=\left(x-x_{b}\right)^{2}+\left(y-y_{b}\right)^{2}
$$

$$
\mathrm{d}_{\mathrm{c}}^{2}=\left(\mathrm{x}-\mathrm{x}_{\mathrm{c}}\right)^{2}+\left(\mathrm{y}-\mathrm{y}_{\mathrm{c}}\right)^{2}
$$

Equations (8),(9)and(10) can be expanded to get the following equations:

$$
\begin{aligned}
& d_{a}^{2}=x^{2}-2 x_{a}+x_{a}^{2}+y^{2}-2 y_{a}+y_{a}^{2} \\
& d_{b}^{2}=x^{2}-2 x_{b}+x_{b}^{2}+y^{2}-2 y_{b}+y_{b}^{2} \\
& d_{c}^{2}=x^{2}-2 x_{c}+x_{c}^{2}+y^{2}-2 y_{c}+y_{c}^{2}
\end{aligned}
$$

By resolving these equations, we determine the intersection point " $x$ " and " $y$ " of these two equations such as the equation for the value of "Y" and the equation of the value of "X" respectively

$$
\begin{aligned}
& y=\frac{v_{b}\left(x_{c}-x_{b}\right)-v_{a}\left(x_{a}-x_{b}\right)}{\left(y_{a}-y_{b}\right)\left(x_{c}-x_{b}\right)-\left(y_{c}-y_{b}\right)\left(x_{a}-x_{b}\right)} \\
& x=\frac{v_{a}-y\left(y_{c}-y_{b}\right)}{\left(x_{c}-x_{b}\right)}
\end{aligned}
$$

The $X$ and $Y$ values give us a precise position in two dimensions (2D) of our blind node. This model was developed using MATLAB and programmed in the node 2420CA, thus after the nodes was initialized, once the blind node receive packets from at least three anchor nodes, the blind nodes can localize their position using references from the anchors. However, localization cannot be obtained without the signal propagation model.

In order to get the third dimension we will take one of the anchor nodes as the reference node (the origin point in this location) as in Fig.4.1. Then we will put anchor nodes in order to divide this dimension into equal parts $\left(z_{0}, z_{1}, z_{2}, \ldots\right)$ first step is to select the anchor nodes the receives the highest signal strength from the blind node. We will select the highest two nodes, now the blind node is located between these two nodes.

To find the value of $z$ as following:

Given d from equation (8)

To get $l_{0}$ as following:

The relation between the Received Signal Strength and the distance using the equation below: 


$$
\begin{aligned}
& \text { RSSI }[\mathrm{dBm}]=-10 n \log _{10}\left(l_{0}\right)+\mathrm{A}[\mathrm{dBm}] \\
& \left(l_{0}\right)=10^{\frac{(\mathrm{RSSI}[\mathrm{dBm}]-\mathrm{A}[\mathrm{dBm}])}{-10 n}}
\end{aligned}
$$

Where, $\mathrm{A}$ is the received signal strength at one meter distance Note we will take the path loss exponent $\mathrm{n}=2.2$ as [11]

By Pythagoras equation

$$
\begin{aligned}
& z_{r}=\sqrt{\left(l_{0}\right)^{2}-(d)^{2}} \\
& \text { total } z=z_{r}+z_{0}
\end{aligned}
$$

\subsection{1 - Signal Propagation Model}

The free space model is most common signal propagation model used in the Wireless sensor network (WSN).In the free space model the receiver within the radius of communications can receive the packets of data. One possibility to get the node distance from another node is to measure the signal strength of the received data packets (the received radio signal). The main idea behind the received signal strength is that the transmitted power $\left(\mathrm{P}_{\mathrm{t}}\right)$ at the transmitter device directly affects the received power $\left(\mathrm{P}_{\mathrm{r}}\right)$ at the receiving device. According to the frii's transmission equation in free space [9], the signal strength detected decreases quadratically with the distance to the sender

$$
\mathrm{P}_{\mathrm{r}}(\mathrm{d})=\frac{p_{t} G_{t} G_{r} \lambda^{2}}{(2 \pi d)^{2}}
$$

Where

$\operatorname{Pr}_{(\mathrm{d})}=$ Received power at the receiver

$\mathrm{P}_{\mathrm{t}}=$ Transmission Power of sender

$\mathrm{G}_{\mathrm{t}}=$ Gain of Transmitter

$\mathrm{G}_{\mathrm{r}}=$ Gain of Receiver

$\lambda=$ Wavelength

$\mathrm{d}=$ Distance between the sender and the receiver normally

$\mathrm{G}_{\mathrm{t}}=\mathrm{G}_{\mathrm{r}}=1$, in embedded devices.

The majority of the integrated systems operate in a non-linear sight environment (NLOS). Based on experimental data, a general model was developed for the propagation o (NLOS). This model predicts that the mean loss of the path $P_{L}\left(d_{i}\right)[d B]$ when separating the transmitter and the receiver is:

$$
\mathrm{P}_{\mathrm{L}}\left(d_{i}\right)[\mathrm{dB}]=\mathrm{P}_{\mathrm{L}}\left(d_{0}\right)[\mathrm{dB}]+10 n \log _{10}\left(\frac{d_{i}}{d_{0}}\right)
$$

Where $n=$ path loss exponent

$\mathrm{PL}\left(d_{0}\right)=$ path loss at known reference distance $d_{0}$ 


$$
n=\frac{\mathrm{P}_{\mathrm{L}}\left(d_{i}\right)-\mathrm{P}_{\mathrm{L}}\left(d_{0}\right)}{10 \log _{10}\left(\frac{d_{i}}{d_{0}}\right)}
$$

\section{2 - Triangulation}

Triangulation is used when estimating the direction of the node rather than the distance, as it is the case in the AOA systems. Node positions in this case are calculated using the trigonometry laws of the sin and cos.

\section{3 -Maximum Likelihood - Estimation (Multilateration and Multiangulation )}

This method is to estimate the maximum likelihood (ML). The position is appreciated by reducing the differences between measured distances and estimated distances. This way defines the nodes with known positions as beacon and those with unknown positions of unknown nodes. The aim is to assess the positions of the largest possible number of unknown nodes in a fully distributed manner. The beacon nodes broadcasts their positions to neighbors. The neighboring nodes are with unknown location measure their separation from their neighbors and use the broadcast beacon positions to calculate their own positions. Once an unknown node calculates its position, it turns to a beacon and broadcasts its estimated position for the other neighboring unknown nodes, allowing them to calculate their positions. This process is repeated until all the unknown nodes that meet the requirements of multilateralism have an appreciation of their position. This process is defined as the iterative multilateration of atomic multilateration, which are used as their primary primitive.

\section{Experimental Test Bed Enviroment}

The test bed environment is shown in the following Fig.6. Our experiment setup used the indoor and outdoor environment of FAYOUM UNIVERSITY Faculty of Engineering Department of Electronics and communication. The test bed consists of four anchor sensor nodes, a base station, and a laptop. All the nodes either the anchor or the blind node are deployed at the test bed. The (base station) sink node is usually attached to the laptop.

\section{Experimental Results}

First we will assume that the value of the propagation path loss exponent is the same as value calculated in Awka, Anambra State, Southeastern Nigeria $n=2.2$ [11] now we will examine different localization algorithms after in order to fine the blind node location $(\mathrm{x}, \mathrm{y})$

\section{. The first algorithm}

First algorithm shown in Fig.7 shows location calculation of blind node in a grid followed by the equations of the algorithm. A, B, C, D is the reference points and $\mathrm{M}$ is the blind node.

Algorithm 1 use equations (23),(24) as the final $x, y$ values for blind node : 


$$
\begin{aligned}
& x=0.5\left[\mathrm{x} 1+\mathrm{x} 2-0.5 \frac{\left(\mathrm{d} 2^{2}+\mathrm{d} 4^{2}\right)-\left(\mathrm{d} 2^{2}-\mathrm{d} 3^{2}\right)}{2(\mathrm{X} 1-\mathrm{X} 2)}\right] \\
& \mathrm{y}=0.5\left[\mathrm{y} 1+\mathrm{y} 2-0.5 \frac{\left(\mathrm{d} 1^{2}+\mathrm{d} 2^{2}\right)-\left(\mathrm{d} 3^{2}-\mathrm{d} 4^{2}\right)}{2(\mathrm{y} 1-\mathrm{y} 2)}\right]
\end{aligned}
$$

Fig. 8 shows results and values of parent- RSSI in the interface program from the health section of the program. Fig.9 shows the error distribution when using algorithm 1 and the positions of blind node that causes error. The blind node sends the signal to the wrong anchor nodes which gives wrong location.

\section{The Second algorithm}

Trilateration method is to estimate the location of node based on simultaneous range measurements from three reference nodes [5]. An estimated position $\left(x_{e}, \mathrm{ye}_{\mathrm{e}}\right)$ is needed to begin the algorithm and at least three reference points $\left(x_{i}, \mathrm{y}_{\mathrm{i}}\right)$ the modification $(\Delta \mathrm{x}$, $\Delta y)$ used in iteration of $\left(x_{e}, y_{e}\right)$ can be established using matrix computation with the following equations.

$$
\begin{aligned}
& \Delta=\left(\mathrm{M}^{\mathrm{T}} \mathrm{M}\right)^{-1} \mathrm{M}^{\mathrm{T}} \mathrm{f} \quad \text { or } \quad \Delta=\left[\begin{array}{l}
\Delta \mathrm{x} \\
\Delta \mathrm{y}
\end{array}\right] \\
& \mathrm{M}=\left[\begin{array}{ccc}
\frac{(\mathrm{x} 1-\mathrm{xe})}{\sqrt{(\mathrm{x} 1-\mathrm{xe})^{2}+(\mathrm{y} 1-\mathrm{ye})^{2}}} & \frac{(\mathrm{y} 1-\mathrm{ye})}{\sqrt{(\mathrm{x} 1-\mathrm{xe})^{2}+(\mathrm{y} 1-\mathrm{ye})^{2}}} \\
\frac{(\mathrm{xi}-\mathrm{xe})}{\sqrt{(\mathrm{xi}-\mathrm{xe})^{2}+(\mathrm{yi}-\mathrm{ye})^{2}}} & \frac{(\mathrm{yi}-\mathrm{ye})}{\sqrt{(\mathrm{xi}-\mathrm{xe})^{2}+(\mathrm{yi}-\mathrm{ye})^{2}}}
\end{array}\right] \\
& f=-\left[\begin{array}{c}
d_{1}-\sqrt{\left(x_{1}-x_{e}\right)^{2}+\left(y_{1}-y_{e}\right)^{2}} \\
\vdots \\
d_{i}-\sqrt{\left(x_{i}-x_{e}\right)^{2}+\left(y_{i}-y_{e}\right)^{2}}
\end{array}\right] \\
& \mathrm{x}_{\mathrm{e}}=\mathrm{x}_{\mathrm{e}}+\Delta \mathrm{x}, \mathrm{y}_{\mathrm{e}}=\mathrm{y}_{\mathrm{e}}+\Delta \mathrm{y}
\end{aligned}
$$

New estimated position $\left(x_{e},\right)$ is obtained from equation (28). The iteration continues until the error is acceptable. By calculating the mean value of the points, the final position is computed. Table.1 shows the results obtained and Fig 10,11, 12 shows the results.

\section{The Third Algorithm}

This method finds the location of the blind node on the intersection of three circles centered at anchor nodes (reference nodes) .Using equations (14),(15)

The result for $X, Y$ gives us the accurate position in (two dimension) for the blind node. This Model is improved using MATLAB, hence after initialization of the nodes.

$$
\text { localization error }(L E)=\sqrt{\left(x_{\text {est }}^{i}-x_{a}^{i}\right)^{2}-\left(y_{\text {est }}^{i}-y_{a}^{i}\right)^{2}}
$$


Table.2 shows actual distance and estimated distance with the actual position.Fig.13 shows the actual position and the estimated position of the blind node.

The limited number of nodes available for experiment was taken to see how the density of the anchor affects the fault of localization. In the indoor experiment that was carried out, the localization error for three anchor nodes was $0.33 \mathrm{~m}$. In the outdoor experiment that was carried out, the localization error for three anchor nodes and four anchor nodes for this system is $0.74 \mathrm{~m}$ and $0.56 \mathrm{~m}$ respectively. Thus, it means that with the addition of one anchor node to the three anchor nodes system in the grid, the localization error reduces by $0.18 \mathrm{~m}$. Based on this, we assumed that our anchor $(\mathrm{Na}=$ $3,4,5,6,7)$ will give localization errors ( $\mathrm{Le}=0.74,0.56,0.38,0.20,0.02)$ respectively.

\section{CONCLUSION}

In this paper, a comparison between three different algorithms has been made from the communication field point of view. Our goal is to keep service uninterrupted regardless of the change in the surrounding environment. Using RSSI method and Algorithm 3, we propose failure prediction method to foresight failure before happening. Algorithm 2 gives results with low localization error which means good accuracy but the accuracy depends on the estimated value. The problem of algorithm 3 is that the blind node might not be always in the intersection point of the three circles. We worked on many experimental setups. Fig.13 shows the result from algorithm3. Localization error $(\mathrm{Le})=0.03 \mathrm{~m}$ by algorithm 2 and equal to $0.3 \mathrm{~m}$ by algorithm 3 that is shown in Fig. 12 for indoor experiments and (Le) $=0.74 \mathrm{~m}$ for outdoor experiments . Table 1 shows RSSI values from reference points (anchor nodes). Fig.9 shows Error distributions when using algorithm 1 . We have implemented a system of localization, which uses a trilateration approach in the wireless sensor network. The location accuracy obtained by the system was evaluated. We conclude that the proposed system to work there must be availability of at least three nodes of the anchor within the network, the anchor nodes broadcast packets containing their locations and other parameters of the sensor, and blind node within the broadcast range can always estimate the distance to node anchor, if the node the blind receive packets from three anchors, it can determine its location.

\section{Figures}

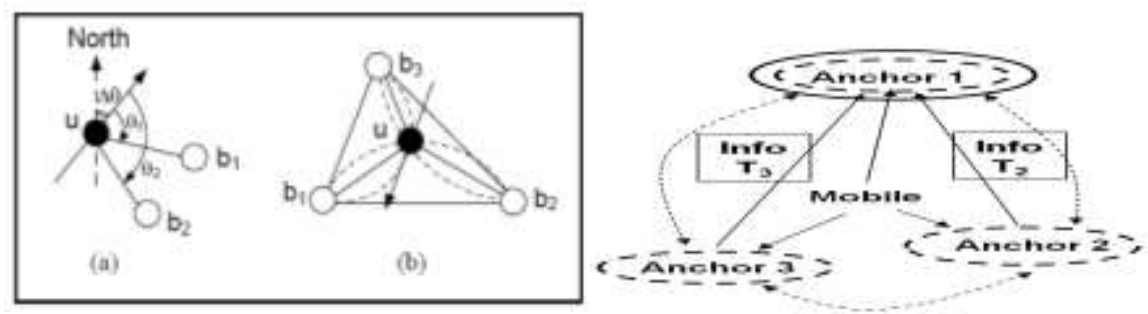

Fig.1: Triangulation in AOA localization:

Fig. 2: Isochronous TOA Estimation and TDOA Estimation

(a) Localization with orientation information; 
(b) Localization without orientation information
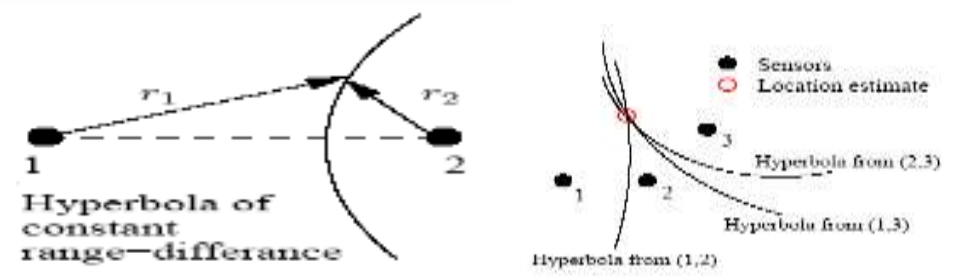

Fig. 3: Hyperbolic Location Theories

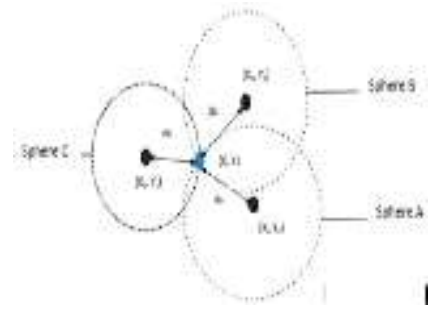

Fig. 4: The three spheres Intersection in 2D

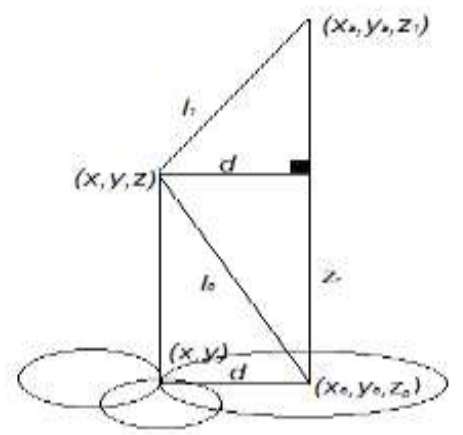

Fig. 4.1: The three spheres Intersection to get 3D

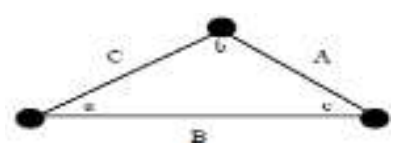

Fig. 5: Triangulation

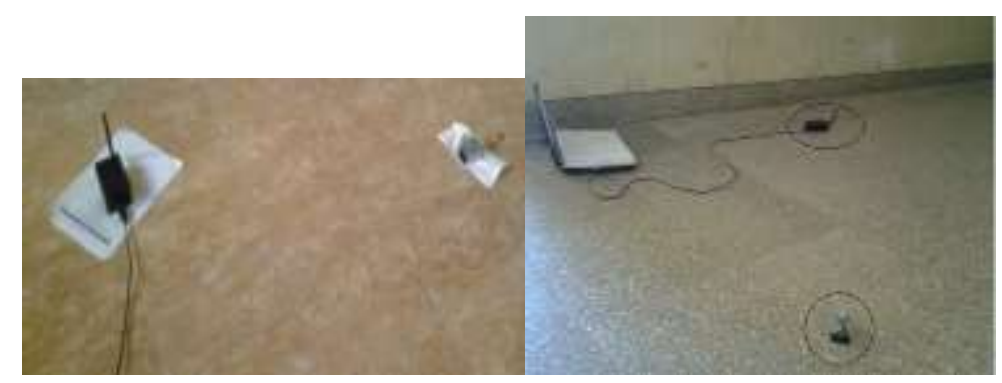

Fig. 6: Test bed environment 


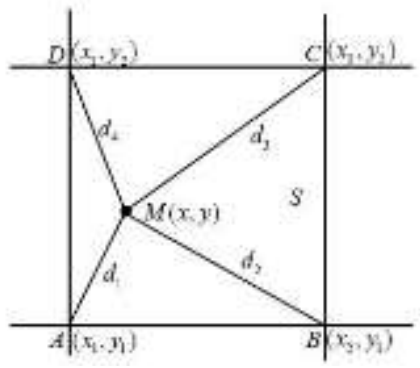

Fig. 7: Calculations position of blind node in a grid.

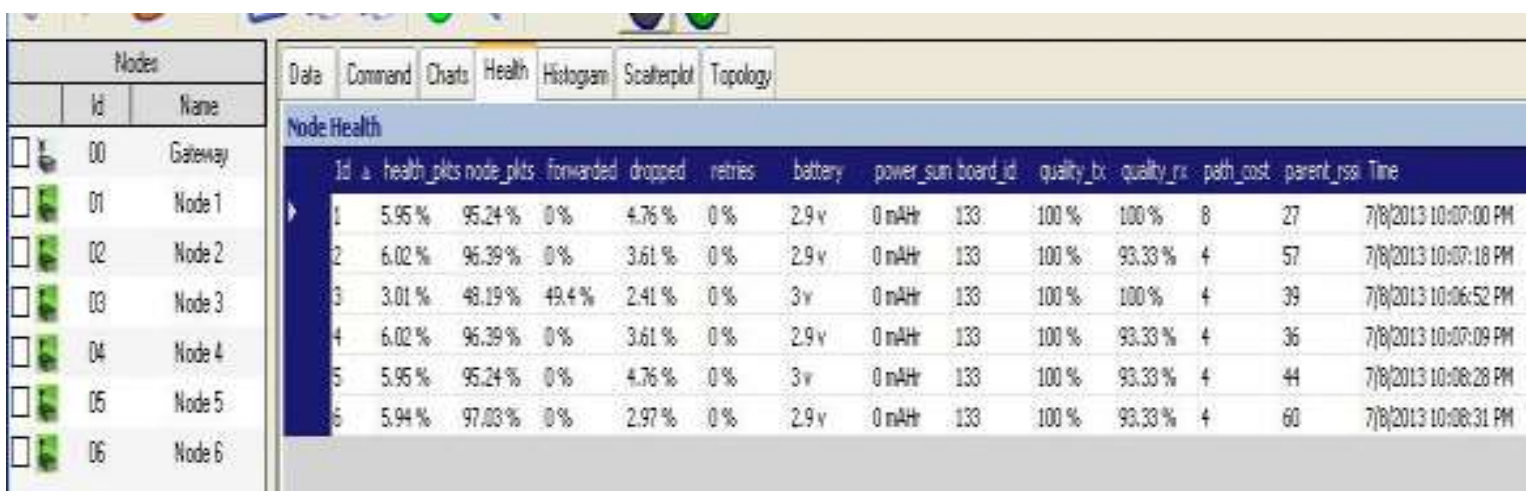

Fig. 8: Result of The first algorithm

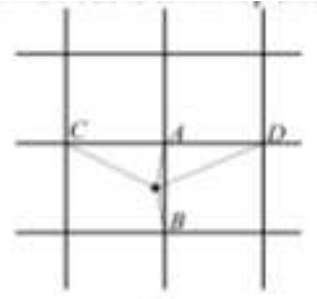

(1)

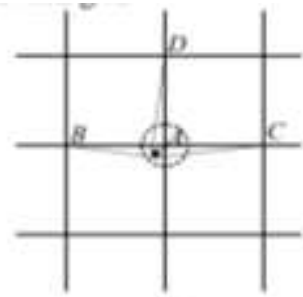

as

Cases of error in algorithm 1

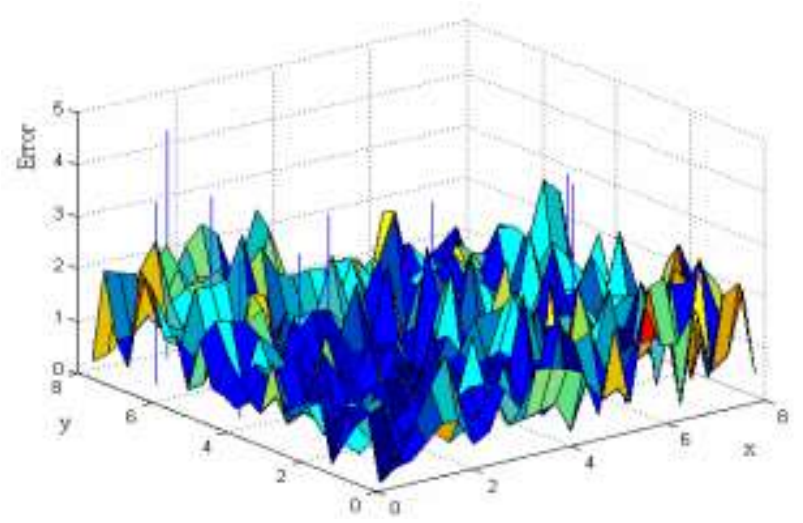

Fig. 9: Error distribution when using algorithm 1 and the positions of blind node that causes error 


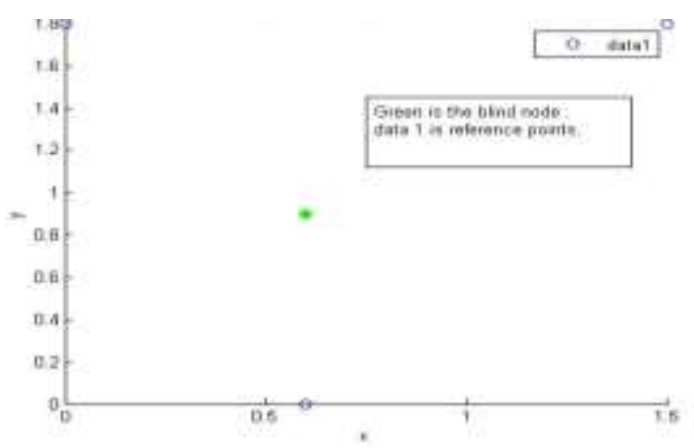

Fig. 10: Three reference points with blind node.

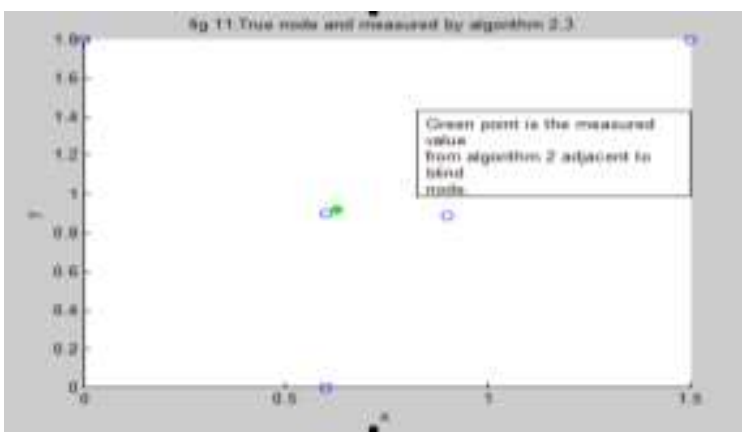

Fig. 11: Here the green node is the measured adjacent to blind node.

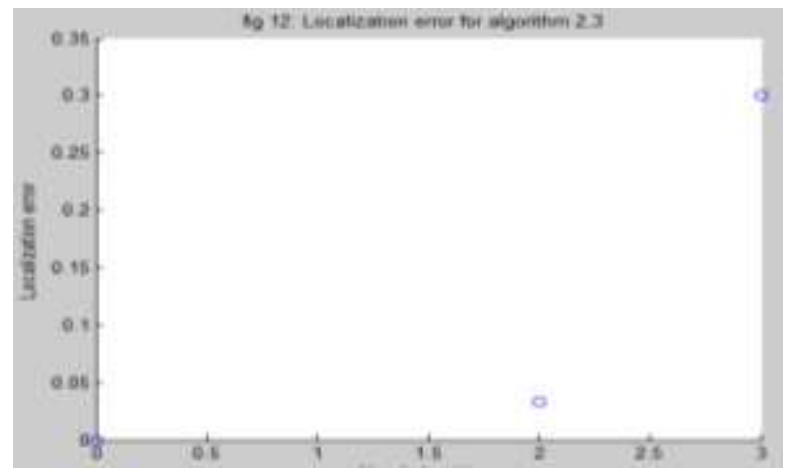

Fig. 12: Localization error (Le) by algorithm 2 and 3.

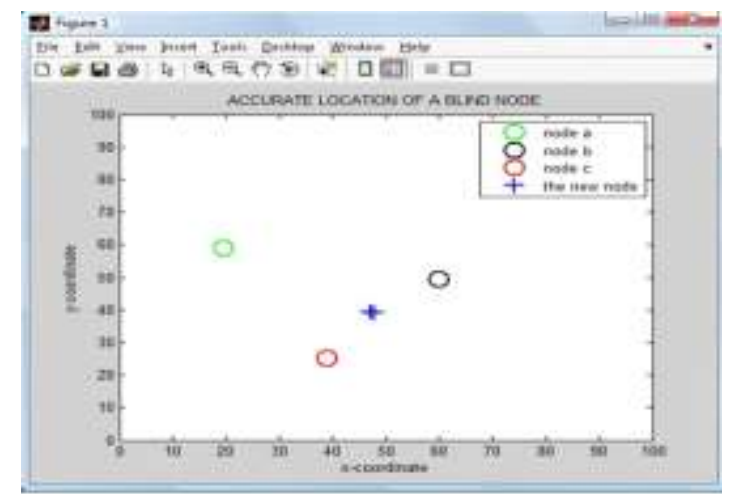

Fig. 13: Estimated position of Blind node 


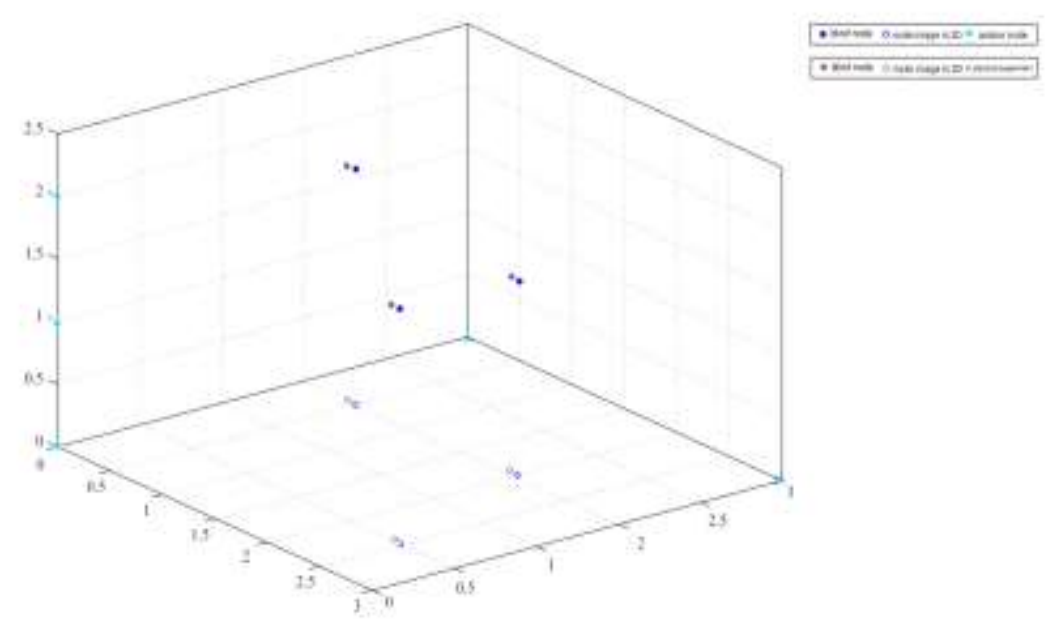

Fig. 14: Estimated position of Blind nodes in 3D

Table 1 RSSI values for algorithm 2.

\begin{tabular}{|c|c|c|c|}
\hline & RSSI values & X & Y \\
\hline RP 1 & $54,45,44$ & .6 & 0 \\
\hline RP 2 & $45,42,45$ & 1.5 & 1.8 \\
\hline RP 3 & $39,43,42$ & 0 & 1.8 \\
\hline
\end{tabular}

Table 2: Node Distance Measurement

\begin{tabular}{|c|c|c|c|}
\hline Anchor & Actual & Estimated & Actual \\
\hline nodes & Distance(m) & Distance(m) & position \\
\hline A & 20.804 & 21 & $(20,60)$ \\
\hline B & 25.072 & 25 & $(60,50)$ \\
\hline C & 16.559 & 17 & $(40,30)$ \\
\hline
\end{tabular}




\section{References}

[1] I.F. Akyildiz and E. cayirci, "A survey on sensor networks," IEEE communication magazine, vol 40, no 8, pp. 102-114(2002),

[2] Jun Zheng and Abbas jamalipour, "Wireless sensor networks: A networking perspective", IEEE communication Magazine (2009),

[3] N. patawari and A.O. Hero III, "Using proximity and quantized RSS for sensors", proc. 2nd ACM international conference on wireless sensor Networks and Application (2003),

[4] E.Elnahrawy, X. Li, and R.P. Martin, "The Limits of Localization using signal Strength: A comparative study", proc. IEEE, (2004) SECON 2004

[5] K.Langendoen and N.Reijers, , "Distributed localization in wireless sensor networks: a quantitative comparison", computer Networks, 43, 2003, 499518(2003)

[6] Dixon, John C, "Suspension Analysis and computational geometry: John wiley and sons limited, (2009).

[7] C. - Y. Chong and S. P. Kumar, "Sensor networks: Evolution, opportunities, and challenges ", "21 ideas for the 21st century", Business week, PP. 78-167 Aug. 301999.

[8] C.Y. chony and S.P.Kumara "sensor networks, Evolution opportunities and challenges", proc. IEEE, vol. 91, no 8, pp.1247-1256,(2003),

[9] Rappaport, Theodore S, "Wireless communications: Principle and Practice". New Jersey, prentice -hall Inc,(1996)

[10] Vijay k Garg, , "wireless communication Networking" san Francisco: Morgan Kauffman publishers(2007)

[11] O.S. Oguejiofor, V.N. Okorogu, A. Abe and B.O. Osuesu, "Outdoor Localization System Using RSSI Measurement of Wireless Sensor Network," International Journal of Innovative Technology and Exploring Engineering (IJITEE), Vol. 2, 2013.

[12] POLITECNICO DI MILANO Polo Regionale di Como Facoltà di Ingegneria dell'Informazione Corso di Laurea Specialistica in Ingegneria Informatica Final Thesis for MSc. Computer Engineering,2010.

[13] Tian He and Chengdu Huang, "Range-Free Localization Schemes for Large Scale Sensor Networks," Proceedings of the 9th annual international conference on Mobile computing and networking, 2003.

[14] Tao Huang , Zhikui Chen, Feng Xia , Cheng Jin , Liang Li," A Practical Localization Algorithm Based on Wireless Sensor Networks". Jul 1, 2007 